\title{
EL ÚLTIMO QUE APAGUE
}

\author{
(THE LAST ONE MUST TURN THE LIGHTS OFF)
}

Raquel Puente García, Dra. Ing. Industrial

Miguel Ángel Rodríguez Lorite, Físico

IPHE

ESPAÑA

Fecha de recepción: 29-XII-98

\begin{abstract}
RESUMEN
Se establece un estudio comparativo entre aquellos sistemas de alumbrado existentes en los edificios de oficinas de antaño y los de ahora, donde la alta tecnología imperante augura un futuro prometedor.
\end{abstract}

\section{SUMMARY}

A comparative study is established between the lighting system existing in old office-buildings and in the modern ones where the prevailing high technogy predicts a promising future.
Ya es parte de nuestra historia "la oficina siniestra", con sus luces macilentas o sus terribles fluorescentes de $38 \mathrm{~mm}$ de diámetro parpadeando sin cesar, donde los esforzados contables aporreaban las Underwood o rellenaban los asientos de los libros a mano. En esos espacios tristes, cuya luz inducía al tedio en sordina con la monotonía de las tareas, no era raro encontrar a la salida el cartelito que recordaba que el último debía apagar la luz.

Pasan los años y con la crisis energética del comienzo de la década de los 70 se impone el celo por ahorrar energía, especialmente en los espacios públicos. El mundo de la luz artificial no ha de quedarse atrás en este proceso y los fabricantes de fuentes de luz se dedican, con especial ahínco, a inventar, reinventar o ensayar nuevas tecnologías para conseguir que sus productos aparecieran en el mercado en condiciones competitivas. De modo que se construyen nuevas lámparas o se mejoran, de manera considerable, las que había, logrando incrementos de eficacia de 50 lúmenes por watio hasta 104 lúmenes por watio en el caso de las de fluorescencia y que, además, van acompañados de mejoras sustantivas en parámetros de calidad de la luz, como, por ejemplo, la reproducción cromática.

Los fabricantes de luminarias no se quedan atrás y, como es lógico, se ensayan nuevos materiales y se diseñan nuevas formas, tanto para las ópticas como para las carcasas y difusores, de modo que los rendimientos mejoren, la luz se dirija adonde debe dirigirse y las luminancias se controlen debidamente, a fin de evitar deslumbramientos molestos.

Téngase en cuenta que la generalización de los ordenadores personales supone un problema añadido en el diseño de iluminación al tener la obligación de resolver, simultáneamente, los problemas visuales en planos horizontal y vertical.

Ofrecer una solución competitiva a las necesidades de los usuarios en estos centros de trabajo exige, además, un estudio de costes acabado, donde se valoren con precisión todos y cada uno de los aspectos de la instalación, su saneamiento, consumo, amortización, etc., etc., más aún cuando es el fabricante de luminarias, en definitiva, quien asume la responsabilidad de comercializar el producto.

En esta carrera por la mejora tecnológica participan, asimismo, los fabricantes de equipos auxiliares. De los $50 \mathrm{~Hz}$ tradicionales se pasa a trabajar con $28 \mathrm{kHz}$ e incluso con $45 \mathrm{kHz}$,reduciendo los tamaños sensiblemente, logrando un control del flujo luminoso con la regulación, con menores consumos de energía reactiva e, incluso, al trabajar con frecuencias tan elevadas, se llega a prescindir 
de los condensadores, visto los altos factores de potencia conseguidos. Y todo esto en tamaños mucho más reducidos y con vidas medias considerablemente superiores.

Para conseguir esto la tecnología eléctrica se apoya, de una manera decisiva, en la electrónica, de modo que se consiguen encendidos y reencendidos casi inmediatos, se alcanza el régimen de funcionamiento óptimo (100\% flujo, temperatura de color, etc.) en pocos segundos, se elimina el efecto estroboscópico, etc.

Resultado de todo ello es, por ejemplo, la posibilidad de adquirir, a un precio adecuado, fuentes fluorescentes compactas con casquillo Edison 27, que sustituyen directamente a las tradicionales incandescentes, con una reducción del $80 \%$ en el consumo eléctrico y con cualidades luminosas aceptables.

Pero si el objetivo último era reducir el consumo de energía eléctrica, resultaría chocante mantener la tradición de que "el último que apague" y, por ello, la informática entra a formar parte de las tecnologías que se aplican en el alumbrado de oficinas. Comienza tímidamente controlando aperturas y cierres de circuitos a ciertas horas, pero hoy ya la encontramos plenamente introducida en este ámbito. Cualquier edificio de oficinas que se precie dispone de un sistema de control, manual o por telemando, en el que la radiación infrarroja ha sustituido parte del cableado, mejorando sustantivamente la versatilidad del espacio y su uso. Si el edificio de oficinas es de los llamados "inteligentes", el control manual desaparece y son los detectores de presencia los que informan al ordenador central de la presencia o no de los usuarios, siendo los temporizadores y las fotocélulas los que regulán el alumbrado en función de las previsiones de uso o el mayor o menor aporte de luz natural. Así, es posible utilizar de una forma eficaz la luz, en el lugar que se precise, durante el tiempo necesario y en la cantidad que la tarea requiera. Esto es lo que se denomina Sistema de Control para luminarias inteligentes, multicanal, de control multifuncional, etc.

Las fuentes luminosas empleadas son, lógicamente, las últimas generaciones de la fluorescencia lineal (trifósforos, multifósforos, etc.), fluorescencia compacta, incandescentes y halógenas.

Se pueden diseñar muy distintas escenas luminosas que serán utilizadas cuando se desee, controlar el gasto, detectar cualquier anomalía, en definitiva gestionar la instalación. Pero además se implementa un Sistema de Control y Gestión Central de todas las instalaciones que coexisten
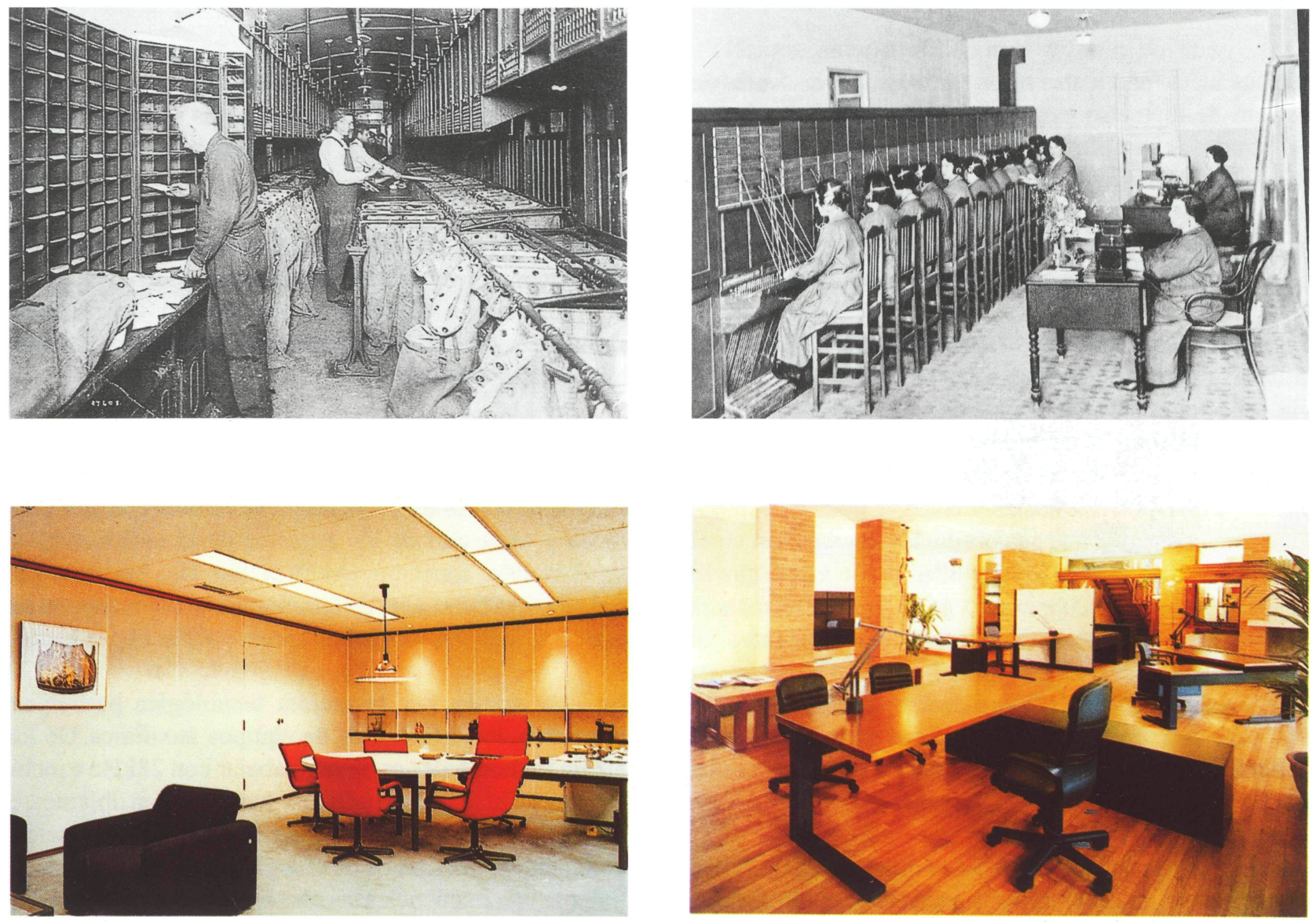

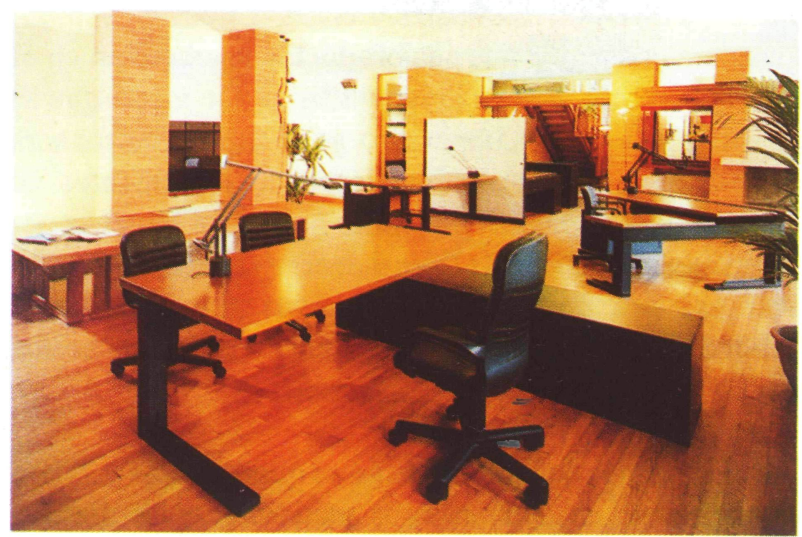

http://informesdelaconstruccion.revistas.csic.es 
en un edificio: podremos tener en cuenta las cargas térmicas disipadas por los equipos de iluminación en el acondicionamiento climático.

El techo tecnológico no es éste; cada vez se integran más desarrollos parciales que podrían conducir a edificios más
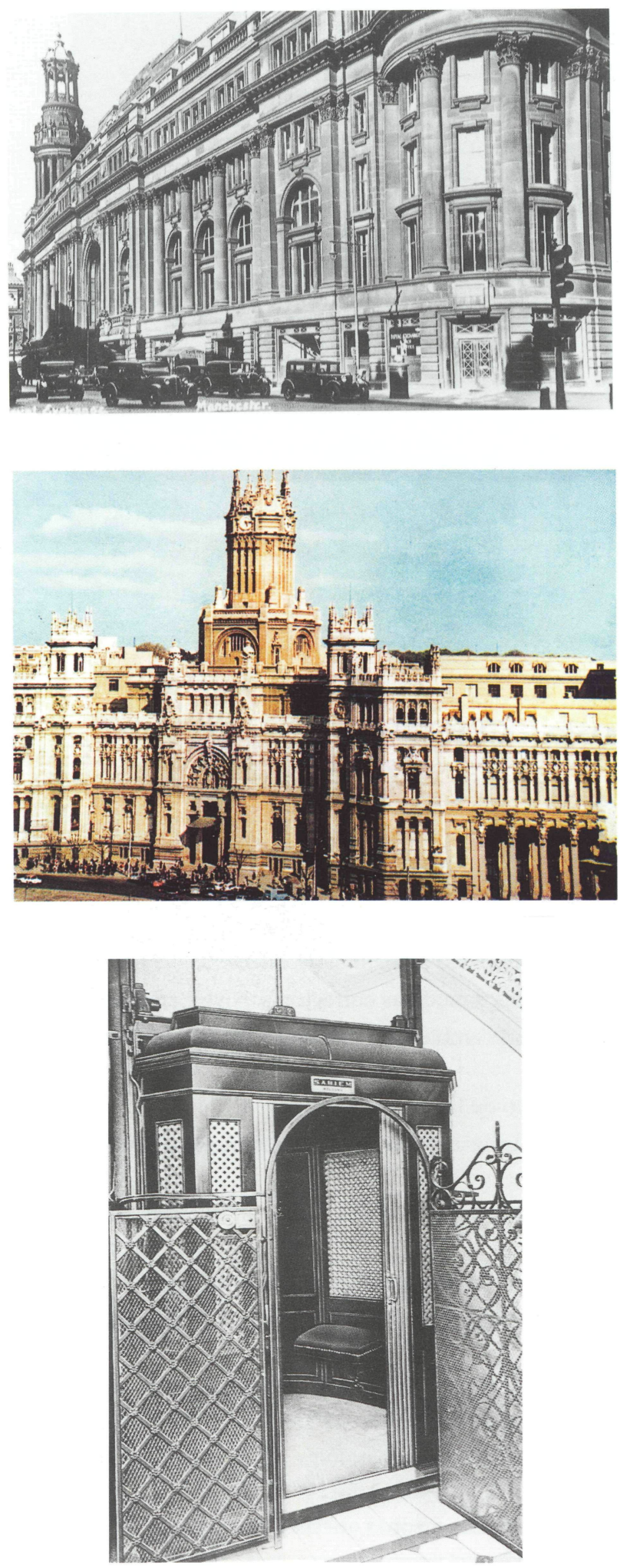

(c) Consejo Superior de Investigaciones Científicas Licencia Creative Commons 3.0 España (by-nc) próximos a la ciencia ficción que a la realidad. Entonces, posiblemente, en ese momento, desaparezca el sujeto y el objeto de la "oficina" y se confunda en la perspectiva histórica al hombre del manguito con el del teléfono móvil. Entonces, las oficinas como espacio de trabajo formarán parte de la arqueología industrial y la etnografía.
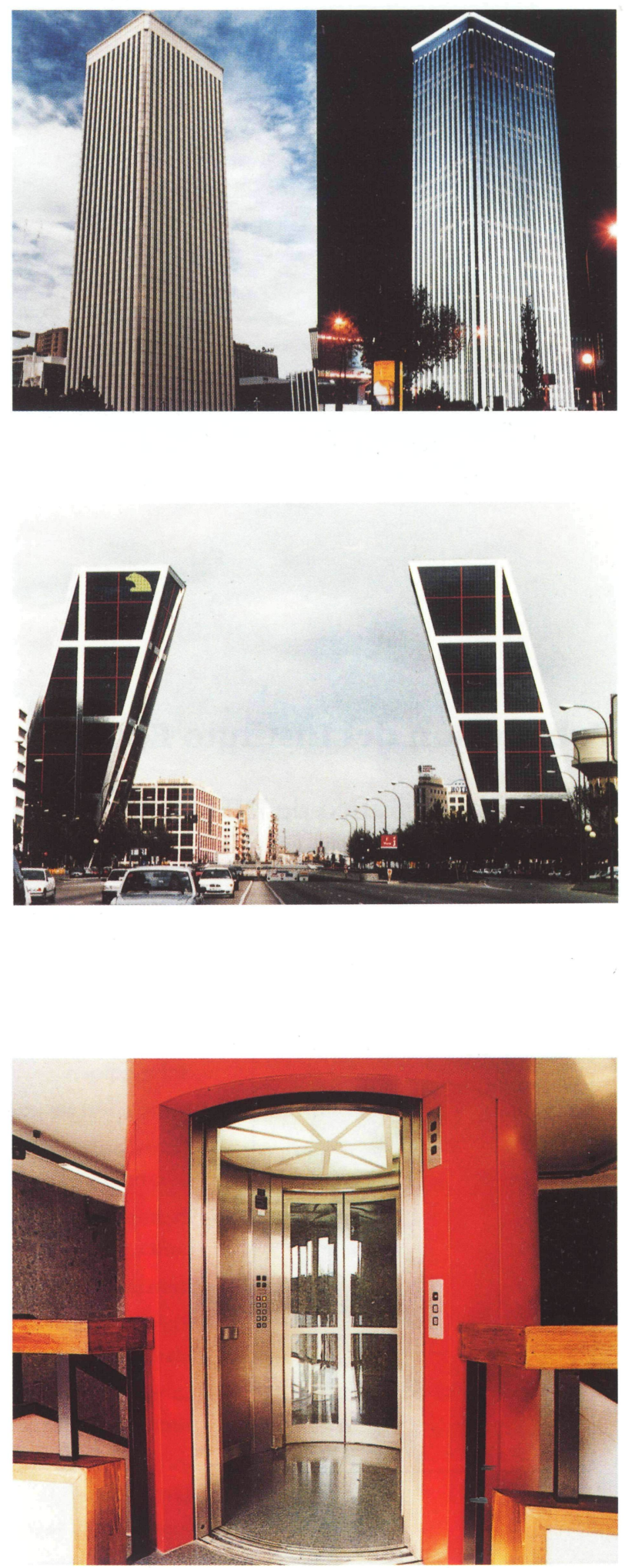

http://informesdelaconstruccion.revistas.csic.es 


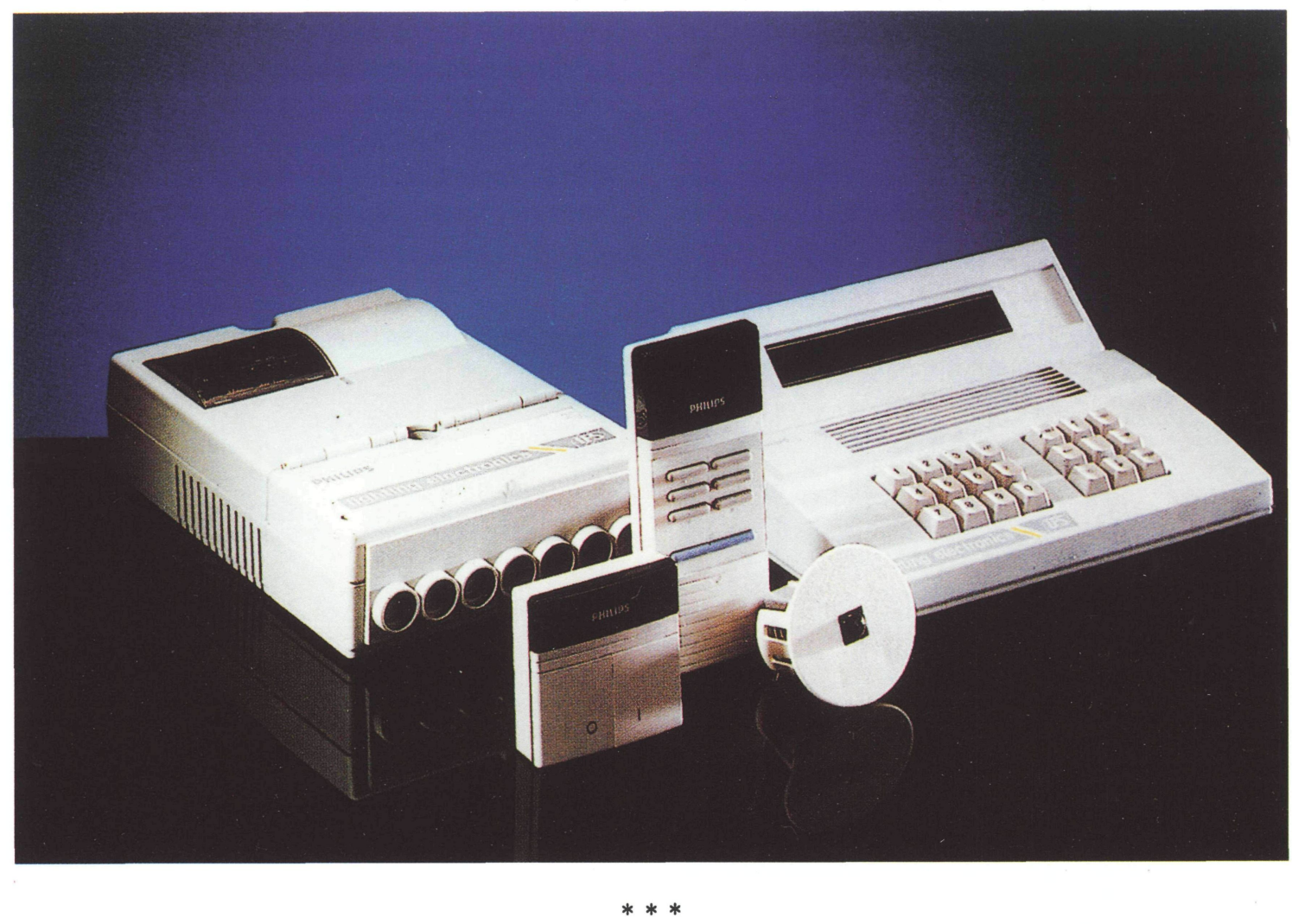

\section{Publicación del Instituto Eduardo Torroja - CSIC}

Número monográfico de INFORMES

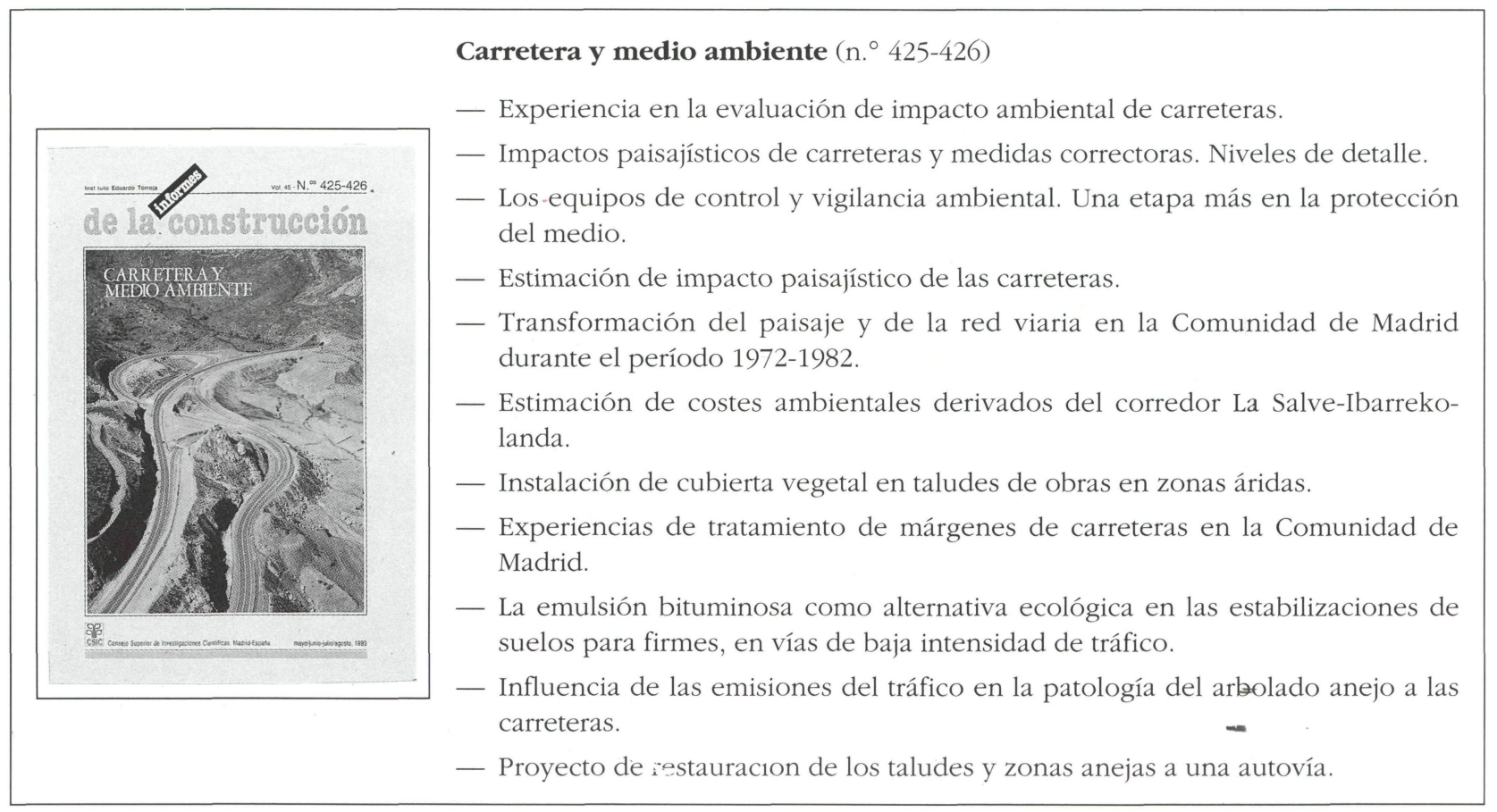

